\title{
JUMPING INSTABILITIES IN THE POST-BUCKLING OF A BEAM ON A PARTIAL NONLINEAR FOUNDATION ${ }^{\star \star}$
}

\author{
Yin Zhang ${ }^{1 \star} \quad$ Kevin D Murphy ${ }^{2}$ \\ $\left({ }^{1}\right.$ State Key Laboratory of Nonlinear Mechanics (LNM), Institute of Mechanics, Chinese Academy of Sciences, \\ Beijing 100190, China) \\ ( ${ }^{2}$ Department of Mechanical Engineering, University of Connecticut, Storrs, CT 06269, USA.)
}

Received 25 November 2011, revision received 17 October 2012

\begin{abstract}
Mode jumping is an instability phenomenon in the post-buckling region, which causes a sudden change in the equilibrium configuration and is thus harmful to structure. The configuration of a partial elastic foundation can directly induce mode coupling from the buckling stage and through the whole post-buckling region. The mode coupling effect due to the configuration of partial foundation on mode jumping is investigated and demonstrated to be an important factor of determining mode jumping. By properly choosing the partial elastic foundation configuration, mode jumping can be avoided.
\end{abstract}

KEY WORDS instability mode jumping tertiary jumping buckling, beam elastic foundation

\section{INTRODUCTION}

When the axially compressive force of a rectangular plate or a beam on an elastic foundation reaches the lowest critical value, the structure experiences supercritical pitchfork bifurcation and deflects to a stable post-buckling configuration if the force is further increased ${ }^{[1,2]}$. The stability of structure in the post-buckling region may be terminated by what is often referred to as secondary buckling or mode jumping ${ }^{[3-13]}$. Mode jumping is a post-buckling instability phenomenon, which is usually marked by a sudden (dynamic) equilibrium configuration change to a new wave pattern of shorter wavelength in loading direction ${ }^{[9,11]}$. Mode jumping is also demonstrated to be the phenomenon that structure reconfigures its post-buckling equilibrium configuration to stay stable ${ }^{[13]}$. By using the theory of singularities and Lyapunov-Schmit procedure, Cheng et al. ${ }^{[12]}$ show that there are forty-five possible postbuckling bifurcation scenarios for a hinged-hinged rectangular plate on a nonlinear elastic foundation and there are several bifurcation scenarios in which mode jumping can occur. Mode jumping is a rather complex phenomenon with many factors playing roles in it. For example, Supple ${ }^{[6]}$ shows that the difference/'nearness' between different buckling loads is an important parameter influencing mode jumping. Therefore, the buckling loads of different modes are usually studied prior to investigating the mode jumping in the post-buckling region ${ }^{[11,13,14]}$. The structure initial imperfection ${ }^{[7,13]}$ and boundary conditions ${ }^{[11]}$ also have significant impact. The initial imperfection ${ }^{[13]}$ or a bracing spring ${ }^{[14]}$ couples the modes from the buckling stage and the boundary conditions determine the mode shape, which in

\footnotetext{
* Corresponding author. E-mail: zhangyin@lnm.imech.ac.cn

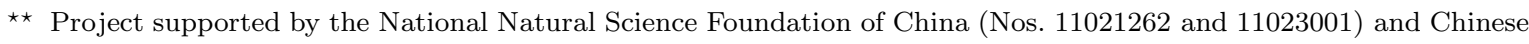
Academy of Sciences (No. KJCX2-EW-L03).
} 
essence affects the modes interaction and, as a result, can significantly influence the mode jumping scenario ${ }^{[7,13]}$. A comprehensive investigation on factors such as the initial imperfection, post-buckling deflection pattern, secondary buckling value and boundary conditions, etc is presented by Uemura and Byon ${ }^{[5]}$. However, the influence of the configuration of a partial elastic foundation, to our best knowledge, is so far not investigated yet. For a beam on a full elastic foundation, when mode jumping occurs, the modes are uncoupled as shown by Supple's theory ${ }^{[6,13]}$. In contrast, the partial elastic foundation configuration couples the modes from the buckling stage and modes are thus coupled when mode jumping occurs. The mode coupling effect on mode jumping is incorporated in Supple's theory of a two degrees-of-freedom (DOF) system ${ }^{[6]}$. However, the mode coupling/interaction in Supple's theory is between two modes. In reality, more than two modes can participate in the structure postuckling deflection as shown in Ref.[13] and this study. The model presented here can incorporate an arbitrary number of modes and the interactions between several models can thus be shown. The elastic foundation here presented is a nonlinear one. From the viewpoint of contact mechanics, the interaction between a beam and its supporting substrate is nonlinear ${ }^{[15,16]}$. The nonlinear elastic foundation model has been used to analyze the buckling propagation and arrest of a pipeline ${ }^{[17]}$, the delamination of sandwich structure driven by buckling ${ }^{[18]}$. Compared with the linear elastic foundation model, the nonlinear elastic foundation model is found to better and more realistically describe the behavior of a system ${ }^{[19]}$.

As the buckled structure still maintains the load-carrying capacity, it has been the source of comfort for a designer to allow for the compressive load larger than its buckling load ${ }^{[5,11]}$. Mode jumping is a post-buckling instability and mode jumping causes a sudden change in the structure equilibrium, which in general is harmful to the structure. Therefore, besides the buckling load, the load of mode jumping is also an important design parameter. The hard film/soft substrate composite structure has been utilized to form the film waviness with a large wave number ${ }^{[20,21]}$, which has been utilized to manufacture highperformance flexural electronics ${ }^{[21]}$. In terms of modeling, the substrate is an elastic foundation and film is a beam/plate ${ }^{[20-22]}$. Waviness, which is also variably called wrinkling, undulation, convolution and ripple ${ }^{[22]}$, is a result of the (post)-buckling of film ${ }^{[20-22]}$. The nominal compressive strain of such a film/substrate system can be as large as $24 \%{ }^{[21]}$, which means severe post-buckling and is also large enough for us to concern about the mode jumping instability of the system. Physically, film may not everywhere be well-bonded with substrate because of the interfacial flaw or the delamination driven by buckling ${ }^{[22]}$ or an external load ${ }^{[23]}$. Furthermore, the out-of-plane bending deformation of a flexural structure can also lead to the separation from its supporting substrate ${ }^{[24,25]}$. The configuration of the partial foundation is often encountered in the real world. For instance, in the stability study of a fluid conveying pipe, the configuration of elastic partial foundation is effectively used as a mechanism to alter and control the system stability ${ }^{[26,27]}$. Some insights into the stability control for the beam-on-elasticfoundation system by altering the elastic partial foundation configuration are revealed in this study. Mode jumping is also shown to be avoidable by properly configuring the elastic partial foundation.

\section{MODEL DEVELOPMENT}

\subsection{Equation of Equilibrium}

Figure 1 shows the schematic diagram of a hinged-hinged beam on a partial nonlinear elastic foundation and the related dimensions. The nonlinear elastic foundation starts from $x=a$ and ends at $x=b$. The beam bending energy $U_{b}$ is given as follows:

$$
U_{b}=\frac{E I}{2} \int_{0}^{L} w_{, x x}^{2} \mathrm{~d} x
$$

where $E I$ is the bending stiffness of the beam; $E$ and $I$ are the beam's Young's modulus and moment of inertia, respectively. $w$ is the beam deflection and $w_{, x x}=\mathrm{d}^{2} w / \mathrm{d} x^{2}$. The work done by compressive force $p$ at the beam ends is given as follows:

$$
W_{p}=\frac{p}{2} \int_{0}^{L}\left(w_{, x}+w_{o, x}\right)^{2} \mathrm{~d} x
$$

where $w_{o}$ is the beam initial imperfection. $K$ is the nonlinear elastic foundation modulus and defined as follows ${ }^{[13]}$ :

$$
K=k_{1}+k_{2}\left(w_{o}+w\right)^{2}
$$




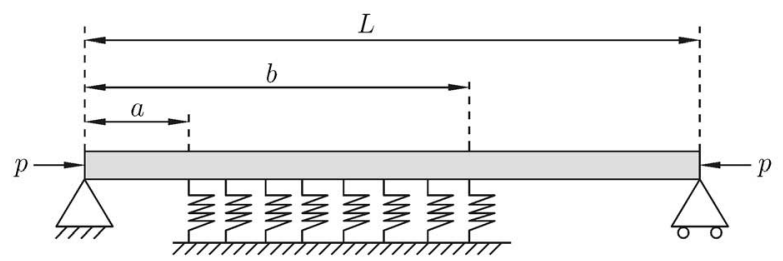

Fig. 1. The schematic diagram and dimensions of the structure.

$k_{1}$ and $k_{2}$ are two given constants. The potential energy stored by the elastic foundation is

$$
\begin{aligned}
U_{f} & =\int_{a}^{b} \int_{0}^{w}\left[k_{1}+k_{2}\left(w_{o}+w\right)^{2}\right]\left(w_{o}+w\right) \mathrm{d} w \mathrm{~d} x \\
& =\int_{a}^{b}\left(k_{1} w_{o} w+\frac{1}{2} k_{1} w^{2}+k_{2} w_{o}^{3} w+\frac{3}{2} k_{2} w_{o}^{2} w^{2}+k_{2} w_{o} w^{3}+\frac{1}{4} k_{2} w^{4}\right) \mathrm{d} x
\end{aligned}
$$

The equilibrium condition requires the system strain energy potential to be stationary, i.e., $\delta\left(U_{b}+\right.$ $\left.U_{f}-W_{p}\right)=0$, the equation of equilibrium is thus obtained as follows:

$\int_{0}^{L}\left[E I w_{, x x x x}+p\left(w_{, x x}+w_{o, x x}\right)\right] \mathrm{d} x+\int_{a}^{b}\left[\left(k_{1}+3 k_{2} w_{o}^{2}\right) w+3 k_{2} w_{o} w^{2}+k_{2} w^{3}+\left(k_{1} w_{o}^{+} k_{2} w_{o}^{3}\right)\right] \mathrm{d} x=0$

Unlike the differential governing equation of a fully supported beam ${ }^{[11,13,14]}$, Eq.(5) now becomes an integrodifferential equation due to the partial foundation configuration. For a hinged-hinged beam, the following boundary conditions hold.

$$
w(0)=0, w_{, x x}(0)=0, w(L)=0, w_{, x x}(L)=0
$$

To non-dimensionalize Eq.(5), the following symbols are introduced

$$
\xi=\frac{x}{L}, \quad W=\frac{w}{L}, \quad W_{o}=\frac{w_{o}}{L}, \xi_{a}=\frac{a}{L}, \quad \xi_{b}=\frac{b}{L}
$$

Now the dimensionless equation of equilibrium is

$$
\int_{0}^{1}\left[W_{, \xi \xi \xi \xi}+\beta_{1}\left(W_{, \xi \xi}+W_{o, \xi \xi}\right)\right] \mathrm{d} \xi+\int_{\xi_{a}}^{\xi_{b}}\left(\beta_{2} W+\beta_{3} W^{2}+\beta_{4} W^{3}+\beta_{5}\right) \mathrm{d} \xi=0
$$

$\beta_{i} s(i=1$ to 5$)$ are defined as follows:

$$
\beta_{1}=\frac{p L^{2}}{E I}, \beta_{2}(\xi)=\frac{k_{1}+3 k_{2} w_{o}^{2}}{E I} L^{4}, \quad \beta_{3}(\xi)=\frac{3 k_{2} w_{o}}{E I} L^{5}, \quad \beta_{4}=\frac{k_{2}}{E I} L^{6}, \quad \beta_{5}(\xi)=\frac{k_{1} w_{o}+k_{2} w_{o}^{3}}{E I} L^{3}
$$

$W$ is assumed to have the following solution form for a hinged-hinged beam on an elastic foundation ${ }^{[13,14]}$,

$$
W=\sum_{j=1}^{N} a_{j} \sin (j \pi \xi)
$$

$a_{j}$ is an unknown constant and $N$ is the mode number. The Galerkin method is used to discretize Eq.(8) and the Newton-Raphson method to solve the nonlinear equation set of $a_{j}{ }^{[13]}$.

The linear part of Eq.(8), which is used to determine the buckling loads (of different modes), is obtained below by truncating the nonlinear parts.

$$
\int_{0}^{1}\left[W_{, \xi \xi \xi \xi}+\beta_{1}\left(W_{, \xi \xi}+W_{o, \xi \xi}\right)\right] \mathrm{d} \xi+\int_{\xi_{a}}^{\xi_{b}}\left(\beta_{2} W+\beta_{5}\right) \mathrm{d} \xi=0
$$




\subsection{Eigenvalues of the Linearized Equation of Equilibrium}

The purpose of finding the eigenvalues of the linearized equation of equilibrium is to tell whether a given equilibrium is stable or not ${ }^{[13]}$. $W$ is now written as the following two parts:

$$
W=W_{\text {eq }}+\bar{W}
$$

$W_{\text {eq }}$ is the equilibrium solution that satisfies Eq.(8). $\bar{W}$ is the (virtual) small deviation from the equilibrium. Plug Eq.(12) into Eq.(8)

$$
\begin{gathered}
\int_{0}^{1}\left[W_{\mathrm{eq}, \xi \xi \xi \xi}+\bar{W}_{, \xi \xi \xi \xi}+\beta_{1}\left(W_{\mathrm{eq}, \xi \xi}+\bar{W}_{, \xi \xi}\right)\right] \mathrm{d} \xi+\int_{\xi_{a}}^{\xi_{b}}\left[\beta_{2}\left(W_{\mathrm{eq}}+\bar{W}\right)+\beta_{3}\left(W_{\mathrm{eq}}^{2}+2 W_{\mathrm{eq}} \bar{W}+\bar{W}^{2}\right)\right. \\
\left.+\beta_{4}\left(W_{\mathrm{eq}}^{3}+3 W_{\mathrm{eq}}^{2} \bar{W}+3 W_{\mathrm{eq}} \bar{W}^{2}+\bar{W}^{3}\right)+\beta_{5}\right] \mathrm{d} \xi=0
\end{gathered}
$$

By taking the linear terms of $\bar{W}$ and using the fact that $W_{\text {eq }}$ satisfies Eq.(8), the linearized integrodifferential equation around the equilibrium is now obtained as follows:

$$
\int_{0}^{1}\left(\bar{W}_{, \xi \xi \xi \xi}+\beta_{1} \bar{W}_{, \xi \xi}\right) \mathrm{d} \xi+\int_{\xi_{a}}^{\xi_{b}}\left(\beta_{2} \bar{W}+2 \beta_{3} W_{\text {eq }} \bar{W}+3 \beta_{4} W_{\text {eq }}^{2} \bar{W}\right) \mathrm{d} \xi=0
$$

This equation is also discretized by the same Galerkin method and $\bar{W}$ is also assumed as

$$
\bar{W}=\sum_{j=1}^{N} b_{j} \sin (j \pi \xi)
$$

$b_{j}$ is unknown constants and Eq.(14) is then discretized as follows:

$$
M B=0
$$

$\boldsymbol{M}$ is an $N \times N$ matrix and each element $M_{i j}$ is given as follows:

$$
\begin{aligned}
M_{i j}= & \int_{0}^{1} \sin (i \pi \xi)\left[\frac{\mathrm{d}^{4} \sin (j \pi \xi)}{\mathrm{d} \xi^{4}}+\beta_{1} \frac{\mathrm{d}^{2} \sin (j \pi \xi)}{\mathrm{d} \xi^{2}}\right] \mathrm{d} \xi \\
& +\int_{\xi_{a}}^{\xi_{b}}\left(\beta_{2}+2 \beta_{3} W_{\text {eq }}+3 \beta_{4} W_{\text {eq }}^{2}\right) \sin (i \pi \xi) \sin (j \pi \xi) \mathrm{d} \xi
\end{aligned}
$$

$\boldsymbol{B}$ is the vector defined as follows:

$$
\boldsymbol{B}^{\mathrm{T}}=\left\{b_{1}, b_{2}, b_{3}, \ldots ., b_{N}\right\}
$$

Now the eigenvalue problem of a system around the equilibrium is to solve the eigenvalues of matrix $M$.

\section{RESULTS AND DISCUSSIONS}

Four cases are selected for the comparison study. The full foundation case ${ }^{[11,13]}$ is selected to account for the comparison. The partial foundation configuration of $a=0, b=0.5 L$ is chosen since this configuration maximizes the participation of the antisymmetric second mode $(\sin (2 \pi \xi))$ in the deflection from the buckling. The configuration of $a=0.2 L, b=0.8 L$ is picked as a symmetric partial foundation study case. The asymmetric configuration of $a=0.2 L, b=0.7 L$ is presented as a comparison to the case of $a=0.2 L, b=0.8 L$ to show how this relatively small asymmetry can dramatically affect the beam post-buckling behavior.

\subsection{Full Elastic Foundation Case of $a=0, b=L$}

The analysis in Ref.[13] focuses more on the tertiary jumping and here the analysis is on the mode coupling. Here $w_{o}$ is set as 0 and $E I=1 \mathrm{~N} \times \mathrm{m}^{2}, k_{1}=1 \mathrm{~N} \times \mathrm{m}^{-2[28,14]}$ and $k_{2}=1 \mathrm{~N} \times \mathrm{m}^{-4[13]}$ for simplicity and comparison reasons. The buckling load for the $m$-th mode is given by Timoshenko ${ }^{[28]}$ as follows:

$$
p_{m}=\frac{\pi^{2} E I}{L^{2}}\left(m^{2}+\frac{k_{1} L^{4}}{m^{2} \pi^{4} E I}\right)
$$


$m$ is a given integer. The buckling loads of different modes versus the beam length are plotted in Fig.2. For the full elastic foundation of $a=0, b=L$, the beam with $L=4.3 \mathrm{~m}$ buckles with the first mode of $\sin (\pi \xi)$, which is marked as a circle in Fig.2. The length of $L=4.3 \mathrm{~m}$ is very close to the transition length of $4.45 \mathrm{~m}^{[13,14,28]}$. The transition lengths marked as triangles in Fig.2 are the crossing points of buckling load curves of different modes. When $L>4.45 \mathrm{~m}$, the buckling load of the first mode becomes larger than that of the second mode; the buckling load of the second mode then becomes the lowest among all buckling loads of modes and beam buckles with the second mode. When $L$ keeps increasing to $7.7 \mathrm{~m}$, the buckling load of the second mode will surpass that of the third; when $L$ increases to $10.86 \mathrm{~m}$, the buckling load of the third mode surpasses that of the fourth and so on and so forth. The buckling load of the beam with $L=4.3 \mathrm{~m}$ on a full elastic foundation is $p=2.42 \mathrm{~N}$, which is marked as a circle in Fig.2. For all cases studied here, $L$ is set as $4.3 \mathrm{~m}$ (thus the beam buckles with the first mode) and mode jumping is expected to occur as from the first mode to the second mode for the full elastic foundation case ${ }^{[11]}$. The state space, which is made up of a control parameter and three modal amplitudes, is introduced as $\left(p, d_{1}, d_{2}, d_{3}\right)^{[13,14]} \cdot p$ is the control parameter of compression force. $d_{1}, d_{2}$ and $d_{3}$ are the amplitudes of $\sin (\pi \xi), \sin (2 \pi \xi)$ and $\sin (3 \pi \xi)$ at a given $p$. At the first buckling point, $\left(p, d_{1}, d_{2}, d_{3}\right)=(2.42,0,0,0)$. Other higher modal amplitudes can also be introduced to the space. For all the cases computed here, the mode number $N$ of the Galerkin method is taken as $N=6$. For the problem analyzed here, the computation shows the contribution of other higher modes to the deflection is trivial and thus not included in the state space defined above.

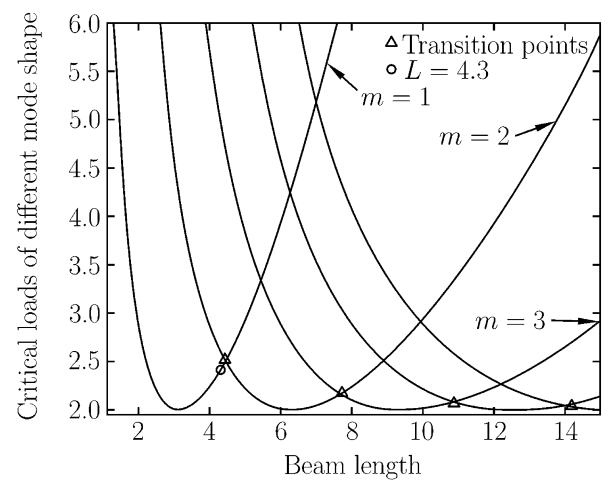

Fig. 2. The buckling loads of different modes for different beam lengths of the full foundation case.

Figure 3(a) shows the path with mode jumping. The solid line indicates the stable equilibrium and dashed one the unstable. The first buckling point is $(2.42,0,0,0)$ marked by a circle in Fig.3(a). The modes remain uncoupled all the time. $u_{1}$ and $u_{2}$ are marked to designate the amplitudes of $\sin (\pi \xi)$ and $\sin (2 \pi \xi)$ at $p=2.82 \mathrm{~N}$, at which mode jumping occurs. $r_{2}$ and $r_{22}$ are marked to designate the amplitudes of $\sin (2 \pi \xi)$ at different compressive loads. When the beam buckles at $p=2.42 \mathrm{~N}$ and enters the post-buckling region, the amplitude of $\sin (\pi \xi)$ begins to increase from zero while the other modal amplitudes still remain zero. At $\left(p, u_{1}, 0,0\right)=(2.82,0.12657,0,0)$, the equilibrium consisting of only the first mode $\sin (\pi \xi)$ becomes unstable and mode jumping occurs. Under the same compressive load $\mathrm{p}$, the equilibrium solution jumps to $\left(p, 0, u_{2}, 0\right)=(2.82,0,0.184,0)$. The stability is told from Eq.(16), which will be discussed later. Mode jumping is the consequence of losing stability in the post-buckling region and snap-through change of equilibrium with a prescribed compressive load. After the system equilibrium jumps to the configuration consisting of only $\sin (2 \pi \xi)$, for unloading, the equilibrium will follow the branch to $\left(p, 0, r_{2}, 0\right)=(2.64,0,0.074,0)$, at which the system loses stability again and jumps to the equilibrium configuration consisting of only $\sin (\pi \xi)$. If unloading continues, the equilibrium consisting of only $\sin (2 \pi \xi)$ is unstable and finally reaches the buckling point of the second mode, marked by $\left(p, 0, r_{22}, ; 0\right)=(2.6,0,0,0)$. From the first buckling to mode jumping and after mode jumping, the modes on this path stay uncoupled. Either mode 1 or mode 2 exists. The post-buckling equilibria as shown in Fig.3(a) exactly match the ones predicted by Supple's theory ${ }^{[7]}$ as shown in Ref.[13]. The detailed derivation of Supple's theory on this particular problem is given 


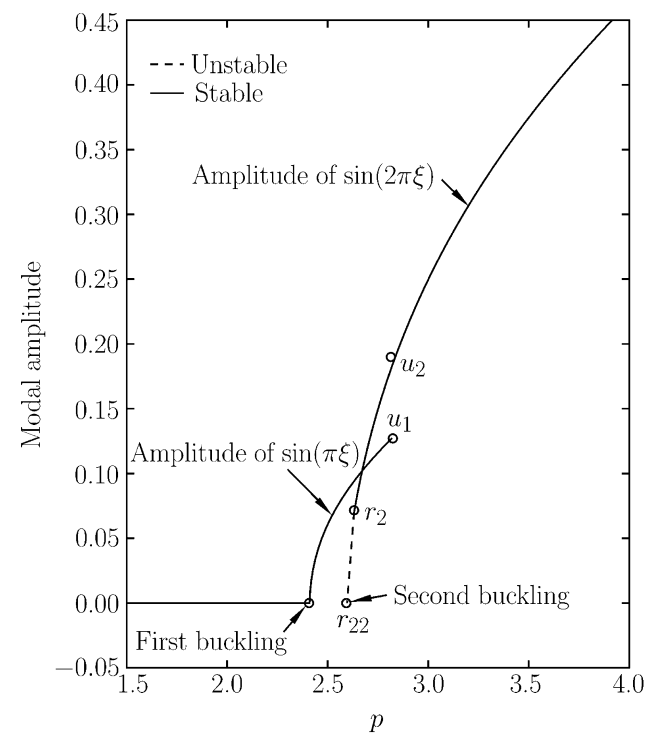

(a)

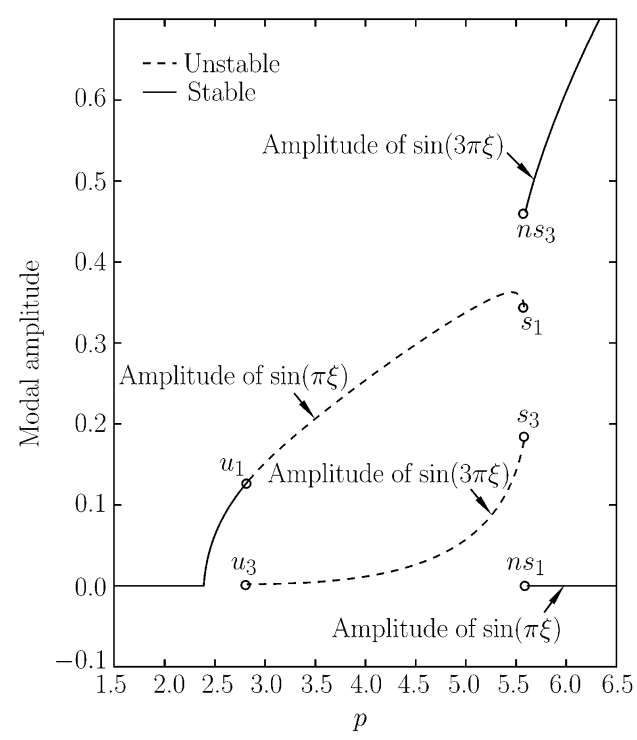

(b)

Fig. 3. (a) The post-buckling path with mode jumping, which is a natural loading path; (b) The post-buckling path with tertiary jumping, which is not a natural loading path.

in Appendix. Supple's theory is in essence an approximate theory, which truncates the higher order differential terms ${ }^{[7,13]}$. Figure 3(b) shows the path with tertiary jumping. $u_{1}$ (the same $u_{1}$ as mentioned above), $s_{1}$ and $n s_{1}$ are marked in Fig.3(b) to designate the amplitudes of $\sin (\pi \xi)$ at different ps. $u_{3}$ (which is zero), $s_{3}$ and $n s_{3}$ are marked to designate the amplitudes of $\sin (3 \pi \xi)$. As shown in Fig.3(a), mode jumping occurs at $\left(p, u_{1}, 0,0\right)=(2.82,0.12657,0,0)$. If this unstable equilibrium is allowed to continue, the third mode begins to participate in the equilibrium at its beginning marked by $u_{3}$. This unstable path continues until $\left(p, s_{1}, 0, s_{3}\right)=(5.58,0.344,0,0.1836)$. At $p=5.58 \mathrm{~N}$, the unstable path becomes stable and then the equilibrium jumps to $\left(p, n s_{1}, 0, n s_{3}\right)=(5.58,0,0,0.4603)$, which is called the tertiary jumping ${ }^{[13]}$. Clearly, the tertiary jumping cannot be reached via natural loading. After tertiary jumping, modes are uncoupled again. In the mode jumping in Fig.3(a) and tertiary jumping in Fig.3(b), the equilibrium solutions presented in the two figures are clearly discontinuous. The continuous solution can be obtained by the path-grabbing algorithm of the arc-length method ${ }^{[13,19]}$. The detailed formulation of the arc-length method for the full foundation case can be found in Ref[13].

In Figs.3(a) and (b), the plots of the stable (solid line) and unstable (dashed line) solutions are based on the eigenvalue study of matrix $\boldsymbol{M}$ in Eq.(16). The eigenvalue is also an indicator of mode jumping ${ }^{[13]}$. Figure 4(a) shows the mode eigenvalues of the path as shown in Fig.3(a). The negative eigenvalues of all modes indicate that the system is stable ${ }^{[13]}$. At $p=2.42 \mathrm{~N}$ of the buckling point of the first mode, the eigenvalue of the first mode marked by an asterisk becomes zero. After entering the post-buckling region, the eigenvalue of the first mode begins to decrease and remains negative, while the eigenvalue of the second mode keeps increasing. $e u_{1}$ and $e u_{2}$ marked by circles in Fig.4(a) are the eigenvalues of the first and second modes before mode jumping at $p=2.82 \mathrm{~N} ; e s_{1}$ and $e s_{2}$ are the eigenvalues of the first and second modes after mode jumping at $p=2.82 \mathrm{~N}$, respectively. $e u_{2}=0$ indicates that the system has lost stability. After mode jumping, $e s_{1}$ and $e s_{2}$ become negative, which means the system has regained stability by mode jumping. Figure 4(b) shows the mode eigenvalues of the path with tertiary jumping as shown in Fig.3(b). $e u_{1}$ and $e u_{2}$ are the same as those in Fig.4(a). $e v_{1}, e v_{2}, e v_{3}$ and $e n v_{1}, e n v_{2}, e n v_{3}$ are the eigenvalues of the first, second and third modes before and after the tertiary jumping. As shown in Fig.3(b), the unstable equilibrium continues rather than mode jumping to regain stability. The eigenvalue of the second mode stays positive until $p=5.58 \mathrm{~N}$, at which the tertiary jumping occurs. The eigenvalues of the second mode before and after the tertiary jumping 


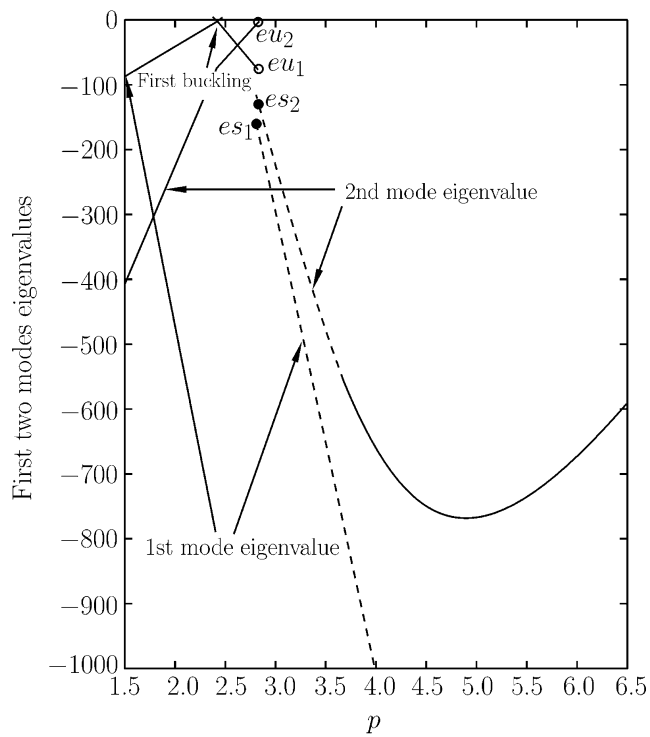

(a)

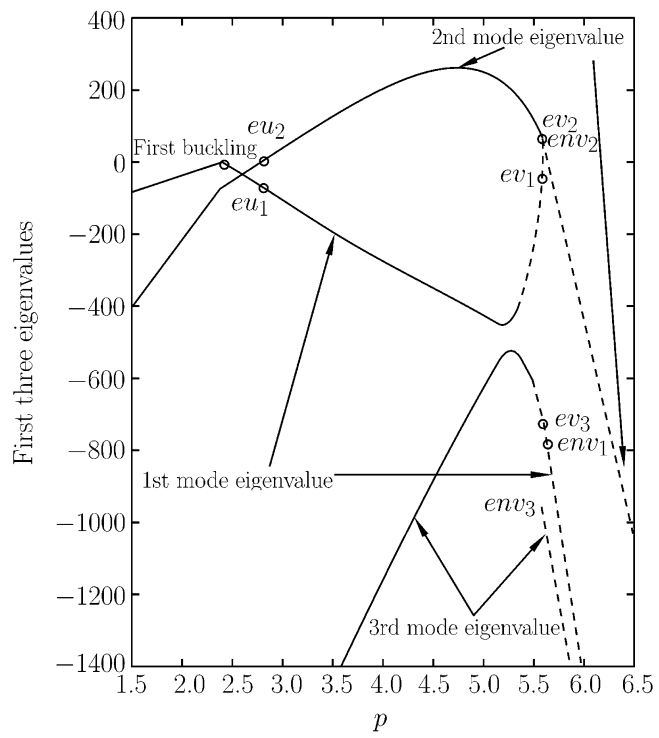

(b)

Fig. 4. (a) Eigenvalues of two different modes on the mode jumping path as shown in Fig.3(a); (b) Eigenvalues of three different modes on the tertiary jumping path as shown in Fig.3(b).

$\left(e v_{2}\right.$ and $\left.e n v_{2}\right)$ as shown in Fig.4(b) stay continuous. However, the eigenvalues of the first and third modes before and after the tertiary jumping (ev $v_{1}$ and $e n v_{1} ; e v_{3}$ and $\left.e n v_{3}\right)$ are discontinuous.

The equilibrium configuration change is a direct result after mode jumping or tertiary jumping. Here the deflection shape is defined as $S=W / W_{\max }=\sum_{j=1}^{N} a_{j} \sin (j \pi \xi) / W_{\max } . W$ is the dimensionless equilibrium as indicated in Eq.(10) and $W_{\max }$ is its maximum value. Figure 5 shows the deflection

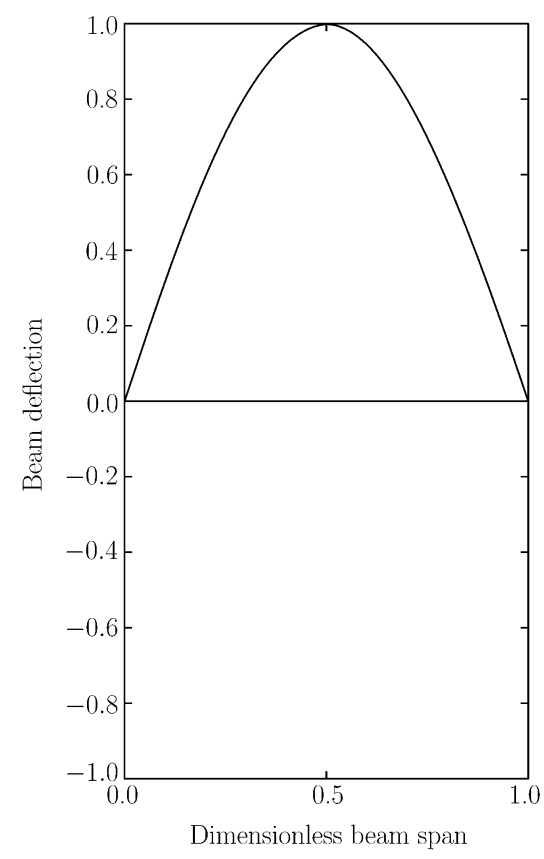

(a)

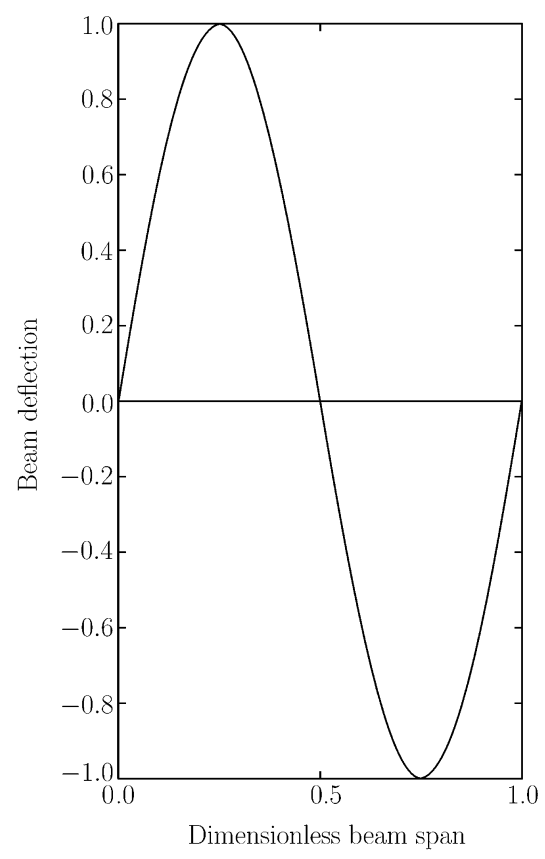

(b)

Fig. 5. Two mode shapes on the mode jumping path of a full foundation case at $\left(p, d_{1}, d_{2}, d_{3}\right)=(2.42,0,0,0)$ and $\left(p, d_{1}, d_{2}, d_{3}\right)=(2.82,0,0.184,0)$, which are before and after mode jumping. 


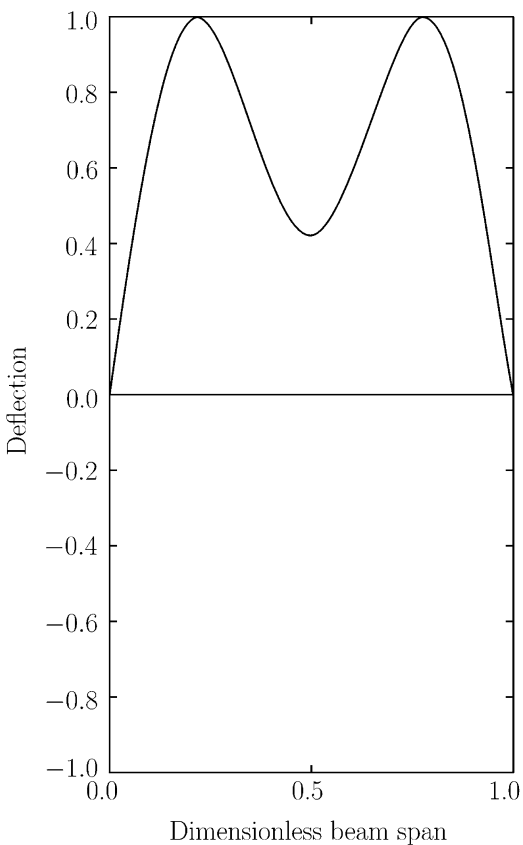

(a)

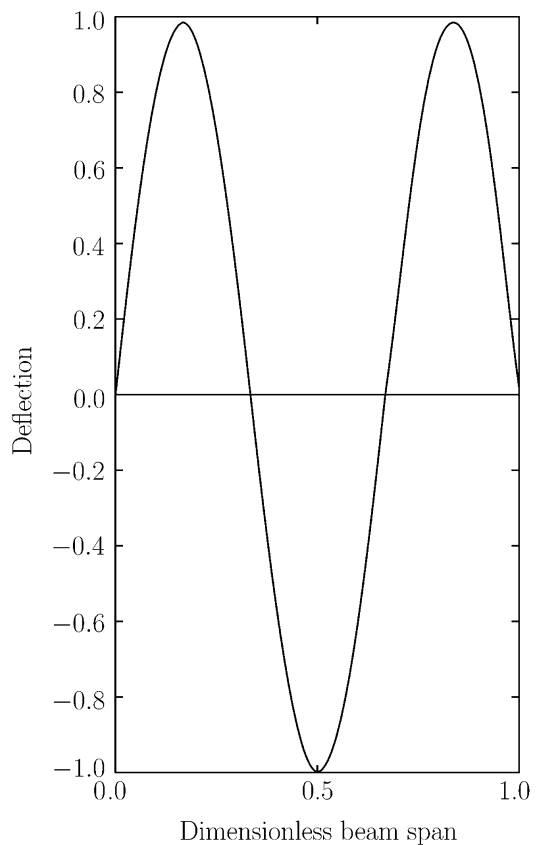

(b)

Fig. 6. Two mode shapes on the tertiary jumping path of full foundation case at $\left(p, d_{1}, d_{2}, d_{3}\right)=(5.579,0.344,0,0.1836)$ and $\left(p, d_{1}, d_{2}, d_{3}\right)=(5.579,0,0,0.463)$, which are before and after tertiary jumping.

shapes before and after mode jumping. Starting from the first buckling to mode jumping, the deflection shape is an uncoupled shape of $\sin (\pi \xi)$ as shown in Fig.5(a). After mode jumping, the mode shape changes to $\sin (2 \pi \xi)$ as shown in Fig.5(b). Figure 6(a) and (b) show the deflection shapes just before and after tertiary jumping, which occurs at (5.58, 0.344, 0, 0.1836). Modes 1 and 3 are coupled before the tertiary jumping. After the tertiary jumping, the modes become uncoupled again and the deflection shape is $\sin (3 \pi \xi)$.

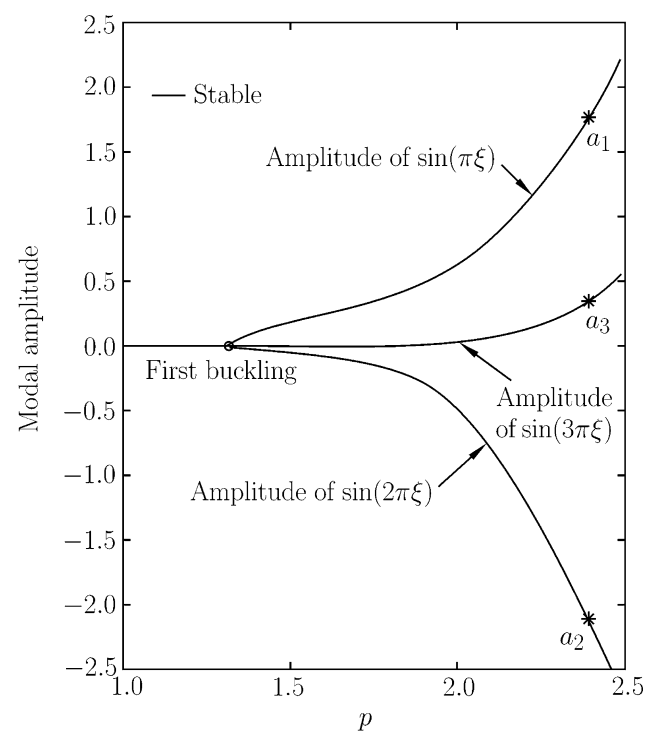

(a)

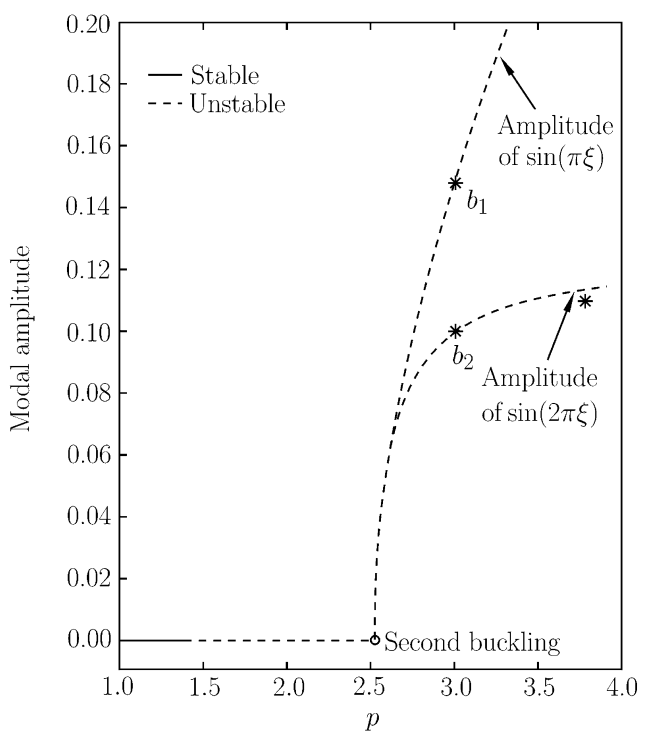

(b)

Fig. 7. (a) The natural loading path solution of partial foundation case of $a=0$ and $b=0.5 L$, which is stable and there is no mode jumping; (b) The other path solution of the partial foundation case of $a=0$ and $b=0.5 L$ case, which is an unstable solution and there is no tertiary jumping. 


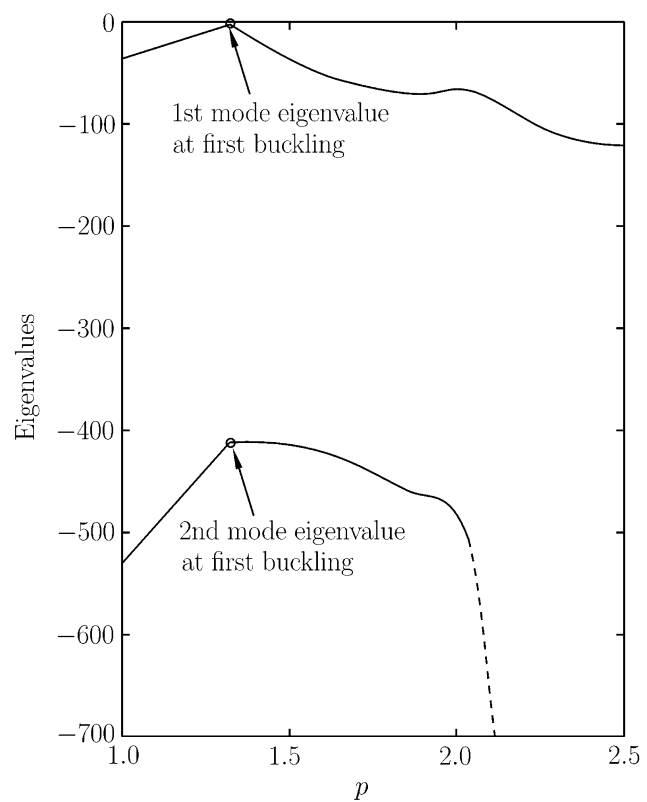

(a)

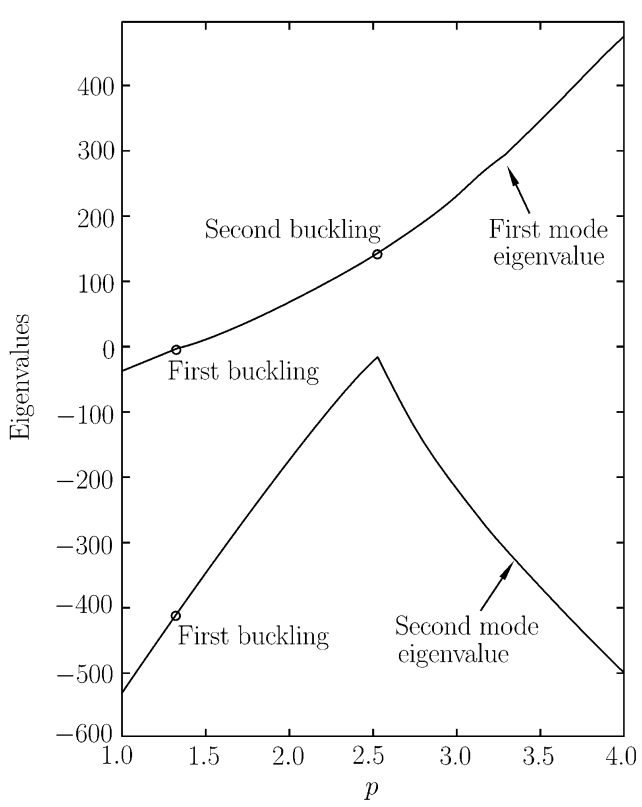

(b)

Fig. 8. (a) Eigenvalues of the two modes on the natural loading (stable) path as shown in Fig.7(a); (b) Eigenvalues of the two modes on the unstable path as shown in Fig.7(b).

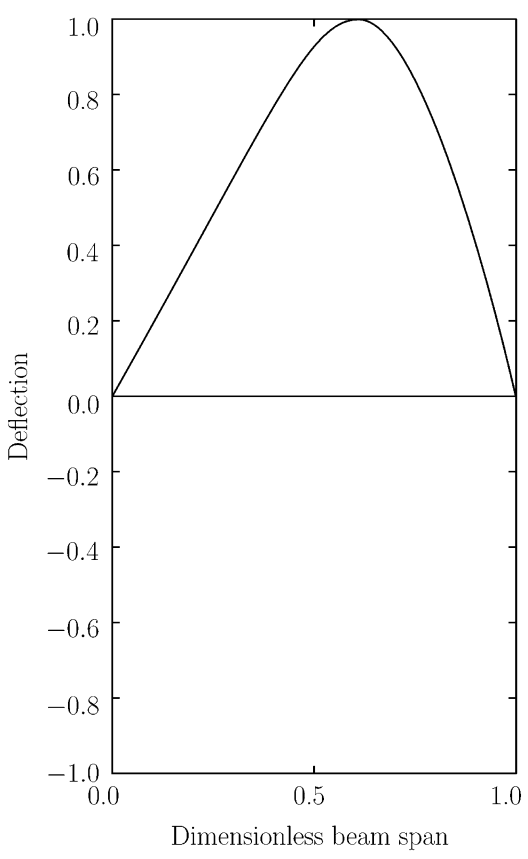

(a)

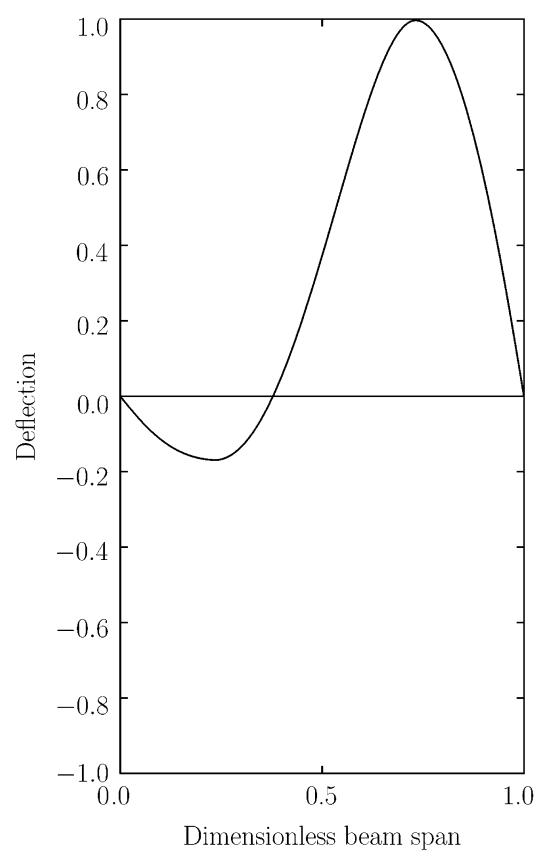

(b)

Fig. 9. Two mode shapes of the stable path as shown in Fig.7(a) at $\left(p, d_{1}, d_{2}, d_{3}\right)=(1.32,0,0,0)$ and $\left(p, d_{1}, d_{2}, d_{3}\right)=$ $(2.4,1.75,-2.1,0.35)$.

\subsection{Partial Foundation Case of $a=0, b=0.5 L$}

It is noticed that for the mode jumping path of a full elastic foundation, the modes $\sin (\pi \xi)$ and $\sin (2 \pi \xi)$ remain uncoupled all the time while the elastic foundation configuration of $a=0$ and $b=0.5 L$ breaks the symmetry and the modes become coupled from the buckling stage. Figure 7(a) shows the natural loading path solution that is stable. It is noticed that the first buckling load decreases dramatically 


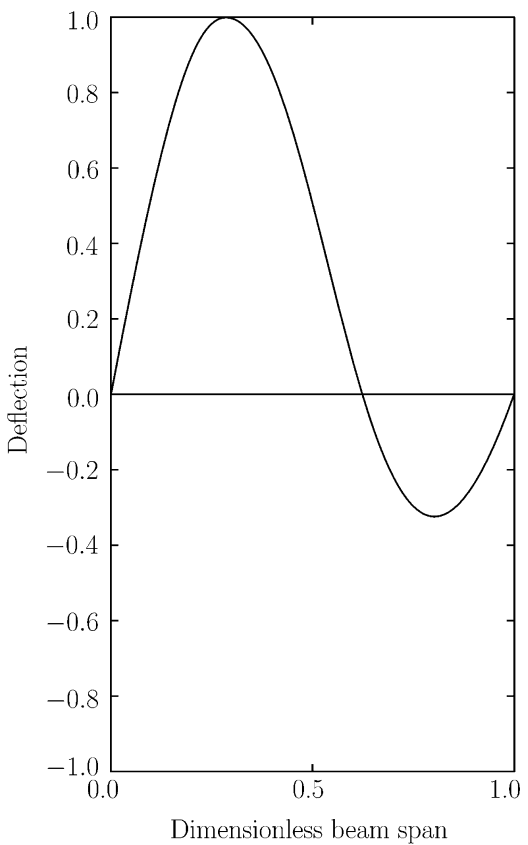

(a)

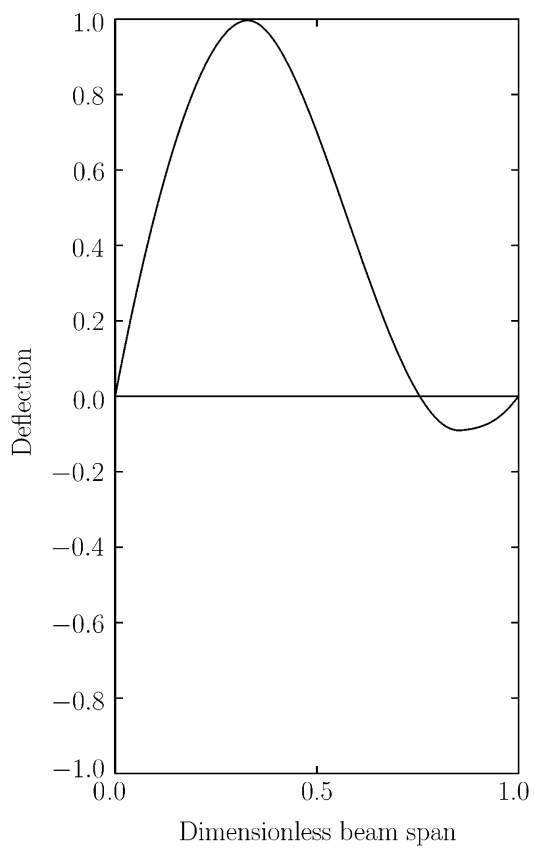

(b)

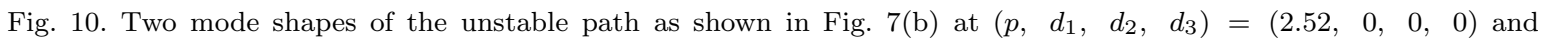
$\left(p, d_{1}, d_{2}, d_{3}\right)=(3,0.1466,0.1,0)$.

to $p=1.32 \mathrm{~N}$ ( $p=2.42 \mathrm{~N}$ for the full elastic foundation case). $\sin (\pi \xi), \sin (2 \pi \xi)$ and $\sin (3 \pi \xi)$ are the three dominant modes participating in the equilibrium deflection shape and coupled from the first buckling. Compared with the full elastic foundation case, there is no mode jumping in Fig.7(a). $\sin (2 \pi \xi)$ participates in the solution from the beginning of the first buckling and gradually becomesdominant. Figure 7(b) shows another path solution around the second buckling that is unstable. There is no tertiary jumping, either. In Fig.7(b), $\sin (\pi \xi)$ and $\sin (2 \pi \xi)$ are also coupled. Figure $8(\mathrm{a})$ is the eigenvalues of mode 1 and 2 in the stable path as shown in Fig.7(a). Figure 8(b) is those in the unstable path as shown in Fig.7(b). The first and second buckling points are marked by circles in Figs.8(a) and (b). Clearly, in both figures the eigenvalues remaincontinuous.

Figure 9 shows the deflection shapes of the stable path as shown in Fig.7(a). The deflection shape of Fig.9(a) is taken immediately after the first buckling point of $(1.32,0,0,0)$. In this deflection shape, $\sin (\pi \xi), \sin (2 \pi \xi)$ and $\sin (3 \pi \xi)$ are coupled and $\sin (\pi \xi)$ dominates at the beginning. Figure $9(\mathrm{~b})$ is the deflection shape at $\left(p, a_{1}, a_{2}, a_{3}\right)=(2.4,1.75,-2.1,0.35) . a_{1}, a_{2}, a_{3}$ are marked in Fig.7(a). It is shown that $\sin (2 \pi \xi)$ is dominant at this stage. Therefore, unlike the full elastic foundation case in which the structure experiences rather sudden and dramatic 'mode jumping', in this partial foundation case, the transition of $\sin (\pi \xi)$ dominance in the deflection shape to $\sin (2 \pi \xi)$ one is smooth. Figure 10 shows the deflection shapes of the unstable path as shown in Fig.7(b). Figure 10(a) is the deflection shape just after the second buckling of $(2.52,0,0,0)$ and Fig.10(b) is one at $\left(p, b_{1}, b_{2}, 0\right)=(3,0.1466,0.1,0) . b_{1}$, $b_{2}$ are also marked in Fig.7(b). Clearly, the contribution of both $\sin (\pi \xi)$ and $\sin (2 \pi \xi)$ to the deflection shapes is significant and the mode coupling effect is strong.

\subsection{Partial Foundation Case of $a=0.2 L, b=0.7 L$}

Figure 11(a) shows the stable path of the solution. The first buckling load increases to $p=2.0 \mathrm{~N}$ $(p=1.32 \mathrm{~N}$ for $a=0, b=0.5 L$ case). $\sin (3 \pi \xi)$ in this configuration has much more contribution to the whole post-buckling deflection than that of the $a=0, b=0.5 L$ case. Still no mode jumping occurs in this partial foundation configuration. The dramatic change happens in the unstable path solution in Fig.11(b). $\sin (\pi \xi), \sin (2 \pi \xi)$ and $\sin (3 \pi \xi)$ all appear in Fig.11(b). Tertiary jumping does not occur, either. In the partial foundation case of $a=0, b=0.5 L$, only $\sin (\pi \xi)$ and $\sin (2 \pi \xi)$ appear. In the full 


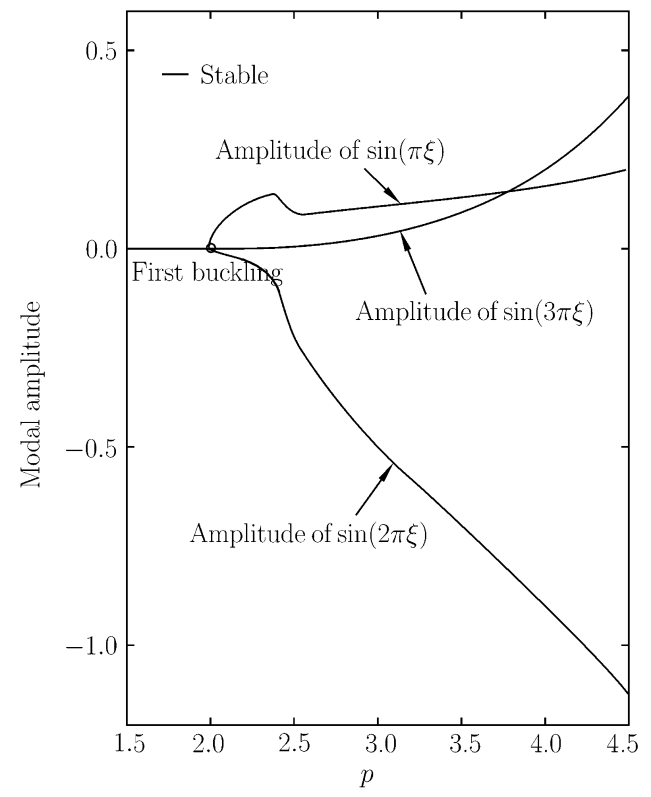

(a)

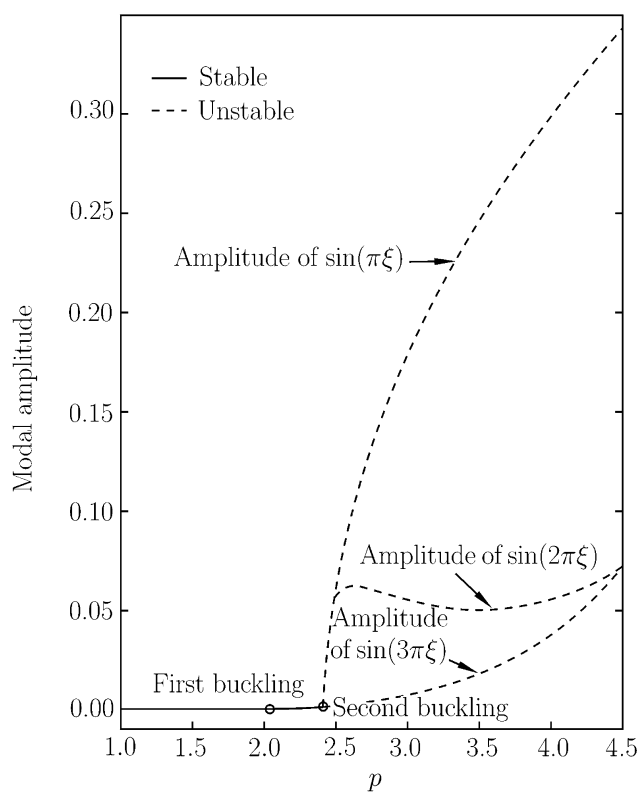

(b)

Fig. 11. (a) The natural loading path solution of a partial foundation case of $a=0.2 L$ and $b=0.7 L$, which is stable and there is no mode jumping; (b) The other path solution of a partial foundation of $a=0.2 L$ and $b=0.7 L$ case, which is an unstable solution and there is no tertiary jumping.

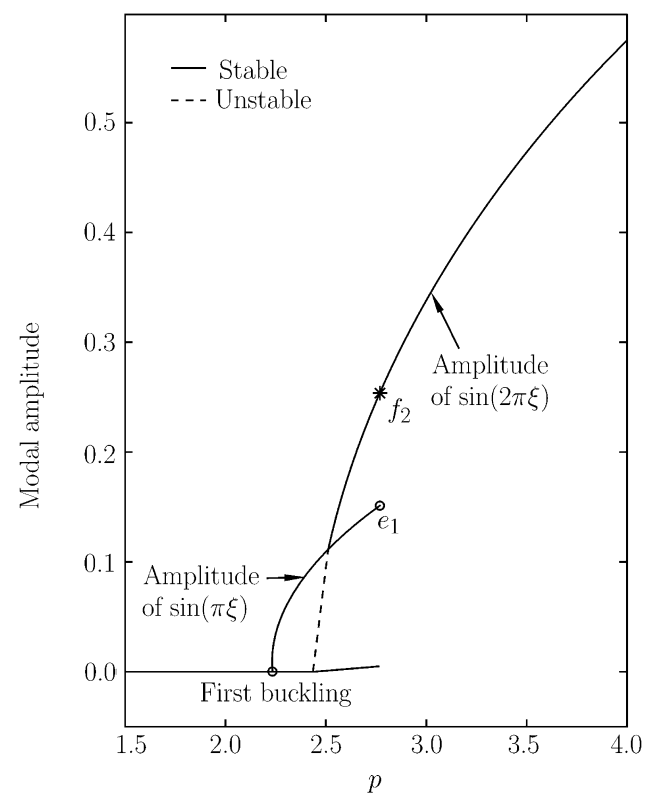

(a)

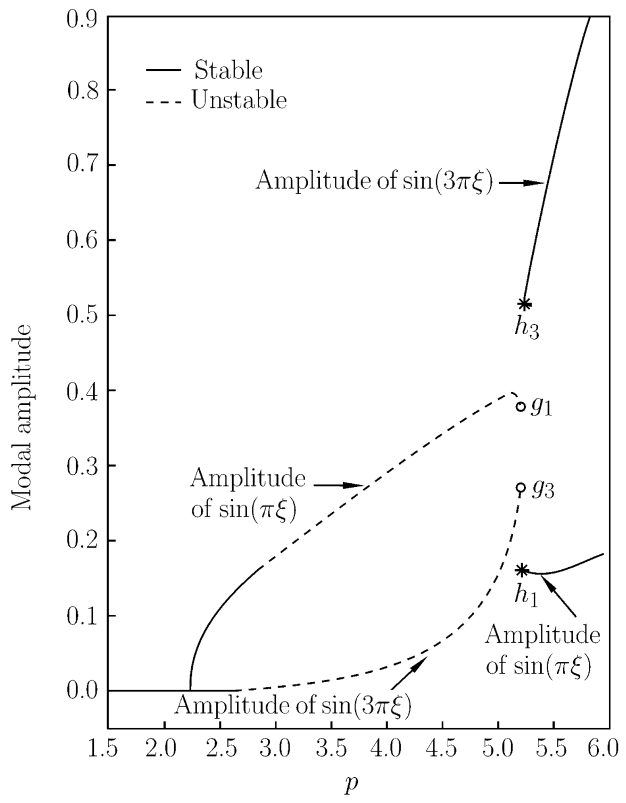

(b)

Fig. 12. (a) The natural loading path solution of partial foundation case of $a=0.2 L$ and $b=0.8 L$, which is stable and mode jumping occurs; (b) The other path solution of the partial foundation case of $a=0.2 L$ and $b=0.8 L$. Tertiary jumping occurs .

foundation case of $a=0, b=L$, only $\sin (\pi \xi)$ and $\sin (3 \pi \xi)$ appear.

\subsection{Partial Foundation Case of $a=0.2 L, b=0.8 L$}

This is a symmetric configuration. Compared with the stable path of $a=0.2 L, b=0.7 L$ case as shown in Fig.11(a), the solution of stable path as shown in Fig.12(a) is quite different. The solution is more like 
the solution of the full foundation case as shown in Fig.3(a). The first buckling is at $p=2.25 \mathrm{~N}(p=2.42$ $\mathrm{N}$ for the full foundation case.) Mode jumping occurs at $p=2.775 \mathrm{~N}(p=2.82 \mathrm{~N}$ for the full foundation case). $e_{1}$ is marked as the amplitude of the first mode before mode jumping and $f_{2}$ as the amplitude of second mode after mode jumping. There occurs mode jumping from $\left(p, e_{1}, 0,0\right)=(2.775,0.152,0,0)$ to $\left(p, 0, f_{2}, 0\right)=(2.775,0,0.251,0)$ compared with the mode jumping in the full foundation case in Fig.3 from $\left(p, u_{1}, 0,0\right)=(2.82,0.12657,0,0)$ to $\left(p, 0, u_{2}, 0\right)=(2.82,0,0.184,0)$. Clearly, the small difference between the configurations of $a=0.2 L, b=0.7 L$ and $a=0.2 L, b=0.8 L$ has huge impact on the stability behavior of the system post-buckling. Figure 12(b) shows the unstable path solution. Tertiary jumping occurs at $p=5.205 \mathrm{~N}(p=5.58 \mathrm{~N}$ for tertiary jumping of the full foundation case). Figure 12(b) also resembles Fig.3(b). $g_{1}, g_{3}$ and $h_{1}, h_{3}$ marked in Fig.12(b) are the amplitudes of the first and third modes before and after mode jumping, respectively. The tertiary jumping in Fig.12(b) from $\left(p, g_{1}, 0, g_{3}\right)=(5.205,0.377,0,0.2724)$ to $\left(p, h_{1}, 0, h_{3}\right)=(5.205,0.1587,0,0.5138)$ occurs compared with the full foundation case of $\left(p, s_{1}, 0, s_{3}\right)=(5.58,0.344,0,0.1836)$ to $\left(p, n s_{1}, 0, n s_{3}\right)=$ $(5.58,0,0,0.4603)$. Therefore, unlike the full foundation case, $\sin (\pi \xi)$ in Fig.12(b) does not die out after tertiary jumping.

In summary, the mode jumping scenarios of full elastic foundation and partial foundation of $a=0.2 L$, $b=0.8 L$ are similar. However, their tertiary jumpings differ significantly.

\section{CONCLUDING REMARKS}

Mode coupling has huge impact on mode jumping and tertiary jumping. Mode jumping occurs as jumping from the mode of $\sin (\pi \xi)$ (symmetric) to $\sin (2 \pi \xi)$ (antisymmetric) for the full foundation case. Both the full foundation case and symmetric partial foundation case $(a=0.2 L, b=0.8 L)$, $\sin (\pi \xi)$ and $\sin (2 \pi \xi)$ remain uncoupled before and after mode jumping. In the asymmetric partial configuration of elastic foundation, which allows the antisymmetric mode like $\sin (2 \pi \xi)$ to participate in the post-buckling deflection, mode jumping can be avoided. As observed from those computations, certain asymmetric partial foundation configurations can facilitate the smooth transition rather than mode jumping. Mode jumping, tertiary jumping and thus dramatic mode shape change can be avoided by configuring asymmetric partial foundation. Generally speaking, mode coupling in the structure postbuckling region depends on the parameters such as $E I, k_{1}, k_{2}, L$, elastic foundation length and location. Here only the influence of the length and location of a partial elastic foundation on mode coupling is presented. The computations also show that tertiary jumping is directly related to mode jumping. As shown in the four cases under discussion, once mode jumping appears/disappears, so will tertiary jumping.

\section{References}

[1] Thompson,J.M.T., Instabilities and Catastrophes in Science and Engineering. John Wiley \& Sons New York, 1982.

[2] Thompson,J.M.T. and Hunt,G.W., Elastic Instability Phenomena. John Wiley \& Sons New York, 1984.

[3] Bauer,L. and Reiss,E.L., Nonlinear buckling of rectangular plates. Journal of SIAM, 1965, 13: 603-626.

[4] Bauer,L., Keller,H.B. and Reiss,E.L., Multiple eigenvalues lead to secondary bifurcation. SIAM Review, 1975, 17: 101-122.

[5] Uemura,M. and Byon,O.I., Secondary buckling of a flat plate under uniaxial compression part 1: theoretical analysis of simply supported flat plate. International Journal of Non-Linear Mechanics, 1977, 12: 355-370.

[6] Supple,W.J., Coupled branching configurations in the elastic buckling of symmetric structural systems. International Journal of Mechanical Sciences, 1967, 9: 97-112.

[7] Supple,W.J., On the change in buckle pattern in elastic structures. International Journal of Mechanical Sciences, 1968, 10: 737-745.

[8] Supple,W.J., Changes of wave-form of plates in the post-buckling range. International Journal of Solids and Structures. 1970, 6: 1243-1258.

[9] Nakamura,T. and Uetani,K., The secondary buckling and post-secondary buckling behaviours of rectangular plates. International Journal of Mechanical Sciences, 1979, 21: 265-286.

[10] Maaskant,R. and Roorda,J., Mode jumping in biaxially compressed plates. International Journal of Solids and Structures, 1992, 29: 1209-1219.

[11] Everall,P.R. and Hunt,G.W., Mode jumping in the buckling of struts and plates: a comparative study. International Journal of Non-Linear Mechanics, 2000, 35: 1067-1079. 
[12] Cheng,C. and Shang,X., Mode jumping of simply supported rectangular plates on nonlinear elastic foundation. International Journal of Non-Linear Mechanics, 1997, 32: 161-172.

[13] Zhang,Y. and Murphy,K.D., Secondary buckling and tertiary states of a beam on a non-linear elastic foundation. International Journal of Non-Linear Mechanics, 2005, 40: 795-805.

[14] Zhang,Y., Liu,Y., Chen,P. and Murphy,K.D., Buckling loads and eigenfrequencies of a braced beam resting on an elastic foundation. Acta Mechanica Solida Sinica, 2011, 24(5): 1-9.

[15] Zhang,Y., Extracting nanobelt mechanical properties from nanoindentation. Journal of Applied Physics, 2010, 107: 123518.

[16] Zhang,Y. and Zhao,Y.P., Modeling nanowire indentation test with adhesive effect. Journal of Applied Mechanics, 2011, 78: 011007.

[17] Chater,E., Hutchinson,J.W. and Neale,K.W., Buckle propagation on a beam on a nonlinear elastic foundation. In Collapse (Edited by Thompson,J.M.T. and Hunt,G.W.), Cambridge University Press, 1983, 31-46.

[18] Bazant,Z. and Grassl,P., Size effect of cohesive delamination fracture triggered by sandwich skin wrinkling. Journal of Applied Mechanics, 2007, 74: 1134-1141.

[19] Youn,S.K., Study of buckle propagation and its arrest on a beam on a nonlinear foundation using finite element method. Computer and Structures, 1991, 39(3/4): 381-386.

[20] Bowden,N., Brittain,S., Evans,A.G., Hutchinson,J.W. and Whitesides,G.M., Spontaneous formation of ordered structures in thin films of metals supported on an elastomeric polymer. Nature, 1998, 393: 146-149.

[21] Khang,D., Jiang,H., Huang,Y. and Rogers,J.A., A stretchable form of single crystal silicon for highperformance electronics on rubber substrates. Science, 2006, 311: 208-212.

[22] Suo,Z. Wrinkling of the oxide scale on an aluminum-containing alloy at high temperature. Journal of the Mechanics and Physics of Solids, 1995, 43: 829-846.

[23] Zhang,Y. and Murphy,K.D., Crack propagation in structures subjected to periodic excitation. Acta Mechanica Solida Sinica, 2007, 20(3): 236-246.

[24] Weitsman,Y., A tensionless contact between a beam and an elastic half-space. International Journal of Engineering Sciences, 1972, 10: 73-81.

[25] Gecit,M.R., Axisymmetric contact problem for an elastic layer and an elastic foundation. International Journal of Engineering Sciences, 1972, 10: 73-81.

[26] Parker,R.G., Supercritical speed stability of the trivial equilibrium of an axially-moving spring on an elastic foundation. Journal of Sound and Vibration, 1999, 221: 205-219.

[27] Elishakoff,I. and Impollonia,N., Does a partial elastic foundation increase the flutter velocity of a pipe conveying fluid? Journal of Applied Mechanics, 2001, 68: 206-212.

[28] Timoshenko,S.P., Theory of Elastic Stability, McGraw-Hill, New York, 1961.

\section{APPENDIX \\ Application of Supple's Theory on a Beam on a Full Elastic Foundation \\ Notation}

$V:$ Total potential energy.

$U_{b}$ and $U_{f}$ : Strain energies of beam (due to bending only) and elastic foundation.

$W_{p}$ : Work done by the axial force.

$p, \delta p$ : Compression and incremental change of compression.

$u_{1}, u_{2}$ : Generalized coordinates representing deformation parameter, here they are modal amplitudes.

$W, \xi$ : Dimensionless transverse deflection of beam neutral axis and coordinate.

$p_{m}, p_{n}$ : Buckling loads of mth and nth modes and $p_{n} \geq p_{m}$ is assumed.

$\Delta p=p_{n}-p_{m}$

$E I, L$ : Beam stiffness and length.

$k_{1}, k_{2}$ : Elastic foundation moduli.

For the beam on the fully supported elastic foundation, the deflection $W$ is assumed to have the following two-coordinate $\left(u_{1}\right.$ and $\left.u_{2}\right)$ solution form

$$
W=u_{1} \sin (m \pi \xi)+u_{2} \sin (n \pi \xi)
$$

$m$ and $n$ are the two mode numbers of the deflection mode shapes. Clearly the above assumption about $W$ can only handle the interaction between two modes. The potential energy $V$ of the whole system is as follows:

$$
V=U_{b}+U_{f}-W_{p}
$$


$U_{b}, U_{f}$ and $W_{p}$ are defined as

$$
U_{b}=\frac{E I}{2 L} \int_{0}^{1} W_{, \xi \xi}^{2} \mathrm{~d} \xi, \quad U_{f}=\int_{0}^{1}\left(\frac{k_{1} L^{3}}{2} W^{2}+\frac{k_{2} L^{5}}{4} W^{4}\right) \mathrm{d} \xi, \quad W_{p}=\frac{p L}{2} \int_{0}^{1} W_{, \xi}^{2} \mathrm{~d} \xi
$$

Substitute equation (20) into Eqs.(21) and (22) and express the potential energy via $u_{1}$ and $u_{2}$ as follows:

$V=\frac{E I \pi^{4}}{4 L}\left(u_{1}^{2} m^{4}+u_{2}^{2} n^{4}\right)-\frac{p L \pi^{2}}{4}\left(u_{1}^{2} m^{2}+u_{2}^{2} n^{2}\right)+\frac{k_{1} L^{3}}{4}\left(u_{1}^{2}+u_{2}^{2}\right)+\frac{k_{2} L^{5}}{4}\left(\frac{3 u_{1}^{4}}{8}+\frac{3 u_{2}^{4}}{8}+1.5 u_{1}^{2} u_{2}^{2}\right)$

The uncoupling conditions defined by Supple ${ }^{[6]}$ are as follows:

$$
u_{1} \neq 0, u_{2}=0 ; \quad u_{1}=0, u_{2} \neq 0
$$

The two coordinates of $u_{1}$ and $u_{2}$ in the post-buckling region can be derived as follows from the two equations given by Supple ${ }^{[6]}$ for the uncoupled mode solution.

$$
\delta p=-\frac{1}{3 !} \frac{V_{1111}}{V_{11}^{\prime}} u_{1}^{2}=\frac{3}{4} \frac{k_{2} L^{4}}{m^{2} \pi^{2}} u_{1}^{2}
$$

and

$$
\delta p=\Delta p-\frac{1}{3 !} \frac{V_{2222}}{V_{22}^{\prime}} u_{2}^{2}=\Delta p+\frac{3}{4} \frac{k_{2} L^{4}}{n^{2} \pi^{2}} u_{2}^{2}
$$

Here a prime on $V$ denotes the partial differentiation with respect to $p$. A subscript on $V$ denotes the partial differentiation with respect to the corresponding coordinate $\left(u_{1}\right.$ or $\left.u_{2}\right)$. For example, $V_{11}=$ $\partial^{2} V / \partial u_{1}^{2}$. For the $L=4.3 \mathrm{~m}$ case, $m=1$ and $n=2 . p_{m}=p_{1}=2.42 \mathrm{~N}, p_{n}=p_{2}=2.6 \mathrm{~N}$. 